Recherches en didactique des langues et des cultures

Les cahiers de l'Acedle

8-1| 2011

Les langues tout au long de la vie: Permanences et évolutions en didactique des langues

\title{
Trajectoires plurielles et représentations identitaires chez les élèves plurilingues
}

\section{Sofia Stratilaki}

\section{(2) OpenEdition}

Journals

Édition électronique

URL : http://journals.openedition.org/rdlc/2231

DOI : $10.4000 /$ rdlc. 2231

ISSN : 1958-5772

Éditeur

ACEDLE

Référence électronique

Sofia Stratilaki, "Trajectoires plurielles et représentations identitaires chez les élèves plurilingues », Recherches en didactique des langues et des cultures [En ligne], 8-1 | 2011, mis en ligne le 01 avril 2001 consulté le 05 mai 2019. URL : http://journals.openedition.org/rdlc/2231 ; DOI : 10.4000/rdlc.2231

Ce document a été généré automatiquement le 5 mai 2019.

\section{(c) $($ ) $\odot \odot$}

Recherches en didactique des langues et des cultures is licensed under a Creative Commons AttributionNonCommercial-NoDerivatives 4.0 International License 


\title{
Trajectoires plurielles et représentations identitaires chez les élèves plurilingues
}

\author{
Sofia Stratilaki
}

\section{Plurilinguisme et représentations identitaires : un maillage des subjectivités}

1 Comment identifier et décrire les représentations des langues et de leur apprentissage chez des apprenants plurilingues? Comment certaines représentations aident-elles les apprenants à construire une identité plurielle et réflexive dont la dynamique se manifeste à travers le jeu des langues? Ces questions ne structurent pas seulement la matière de cette étude, mais aussi une problématique qui concerne plus généralement les disciplines scientifiques et la vie sociale de tous les jours. En effet, sous des appellations variables ( identités trouées, identités fragmentées, identités premières, identités circonstancielles, identités culturelles, identités revendiquées pour ne citer qu'elles) semble se dessiner, dans les divers courants épistémologiques portant sur les rapports aux langues, un large consensus sur la nécessité et l'intérêt qu'il y a aujourd'hui d'étudier la complexité de la construction identitaire en situation de contact de langues (Castellotti \& Moore, 2002). Nous souhaitons, pour notre part, nous interroger sur les liens entre les apprentissages langagiers à l'école et le développement d'une identité plurielle chez des apprenants plurilingues.

Plus particulièrement, nous nous attacherons à mettre en évidence les relations entre biographies langagières et construction d'identités plurilingues en interrogeant des données orales recueillies auprès d'apprenants de langues, en l'occurrence des élèves franco-allemands scolarisés dans un établissement institutionnellement valorisant et réputé comme l'est le lycée de Sarrebruck en Allemagne. Pour ce faire, nous préciserons, de manière relativement succincte, les modalités par lesquelles les représentations, en tant que valeurs, idées ou images, préfigurent ou reconfigurent certains éléments 
constitutifs de l'identité de l'apprenant. Ensuite, nous soulignerons les enjeux que le discours en général, et la description en particulier, mobilisent dans les récits langagiers où se déploie cette identité plurielle et complexe de l'apprenant. Dans cette perspective, nous faisons l'hypothèse que les représentations ne sont pas de simples images stabilisées propres à des sujets ou à des collectivités mais des versions du monde qui apparaissent, sont négociées, éventuellement imposées, transformées, reformulées sans cesse dans les interactions situées entre acteurs sociaux et permettent de comprendre, à différents niveaux, les identités sociales et les comportements linguistiques. En ce sens, la description, en tant que dispositif pertinent pour l'analyse du discours, ne renvoie pas à une réalité externe mais plutôt à la façon dont le locuteur-acteur se confronte à, gère, maintient et transforme son identité, sa vie sociale et communicationnelle. Nous considérons que l'élève suit un itinéraire dynamique dans la construction de son identité, dans la mesure où la biographie langagière n'apparait pas, dans son discours, comme une entité figée mais comme le produit d'étapes successives de démarcation ou d'identification à tels ou tels prototypes, de négociations constantes entre l'image de soimême et celle du locuteur plurilingue, ou même d'une négociation constante entre l'expérience de participation dans des communautés langagières différentes et les projections de soi à l'intérieur de ces communautés. Ce qui signifie, pour nous, qu'une identité plurilingue n'est pas une donnée statique mais qu'elle peut être évolutive, traversée tout au long de la vie par des restructurations en fonction des situations et des relations sociales.

Ce choix découle directement de l'objectif poursuivi depuis quelques années et qui consiste à étudier les représentations du plurilinguisme, des pratiques langagières et des dires des élèves franco-allemands, en particulier la façon dont se dessinent les profils d'apprenants plurilingues dans trois contextes différents d'enseignement bilingue en France et en Allemagne. Deux entrées seront privilégiées dans notre approche. D'une part, nous envisagerons l'apprentissage des langues dans sa dimension diachronique, comme un parcours construit tout au long de la vie, prenant en considération le vécu langagier de l'apprenant en dehors de l'école ou avant celle-ci, c'est-à-dire le capital de relation à l'altérité déjà-là ou en voie de constitution des apprenants. D'autre part, nous adopterons une perspective synchronique pour aborder les compétences langagières à un moment précis dans la vie d'un apprenant. L'accent sera mis à la fois sur l'identité actuelle et sur les identités possibles, définies comme les projections par l'individu de ce qu'il veut être, l'objectif étant de répondre aux questions suivantes: comment les biographies langagières, auxquelles on accède par le biais de l'analyse des récits, mettent en lumière différents parcours de construction identitaire? Comment certaines représentations aident-elles les apprenants à construire une identité plurielle et réflexive dont la dynamique se manifeste à travers le jeu des langues? Et comment les différentes expériences d'apprentissage des langues, à l'école et en dehors de celle-ci, sont susceptibles d'enrichir ou de complexifier les pratiques et les représentations de l'apprenant tout au long de sa vie?

\section{Construire des représentations identitaires : voix/ voies d'interprétation}

4 Pour essayer de répondre à ces questions, précisons d'abord le cadre théorique dans lequel s'inscrit notre recherche. Les recherches européennes et nord-américaines (Pepin, 
2007 ; De Florio-Hansen \& Hu, 2007 ; Deschamps \& Moliner, 2008), dans leur expression en français, en anglais et en allemand, nous proposent un angle de lecture plurilingue particulièrement intéressant pour aborder la question de l'apprentissage et des représentations des langues comme éléments concourant à la construction identitaire des apprenants. Au sein de ce courant de recherches, les attitudes et les motivations des apprenants sont étudiées à l'aide d'un appareil conceptuel qui accorde une large place aux termes de participation et de communautés discursives imaginées. L'imaginaire, envisagé comme un espace créatif et un processus discursif, joue un rôle fondamental dans l'élaboration des stratégies identitaires et d'appropriation des langues, permettant aux élèves d'aller au-delà des pratiques locales, de se projeter dans l'avenir, de concevoir d'autres identités langagières, de nouvelles manières de se voir et de voir le monde. La notion de participation, quant à elle, devient une notion clé pour appréhender l'apprentissage non seulement comme l'intériorisation par l'individu d'un code linguistique mais aussi comme les schèmes d'action ou les processus identitaires à travers lesquels on devient membre d'une communauté discursive, en ce sens que l'on est capable de communiquer, à géométrie variable, dans la (les) langue(s) de la communauté et d'agir selon ses normes, qu'il s'agisse d'une communauté réduite (réseau amical, familial) ou plus vaste (région, pays), occasionnelle (vacances, séjours linguistiques, stages) ou plus durable (école, mobilité transfrontalière). En ce sens, les pratiques langagières, les formes de participation dans l'espace et le temps, peuvent être conçues comme une série d'actes d'identité (de figures identitaires au sens de Goffman, 1973) à travers lesquels les élèves expriment leurs expériences personnelles et leurs aspirations à des rôles sociaux. Dans une telle approche, l'identité ne peut pas être considérée comme simplement "donnée" et ne représente pas non plus un simple contenu traditionnel auquel l'élève doit s'identifier, mais que les élèves et les enseignants participent ensemble, par leur comportement, à la formation de l'identité plurilingue, qui résulte des intentions et des projets plutôt que des conditionnements et des schèmes prescrits.

5 En même temps, le récit langagier, défini en termes de complexe de séquences hétérogènes à orientation narrative dominante, permet de penser le discours à partir de l'ordre de la pluralité, où l'on observe que les séquences peuvent remplir des fonctions multiples, imbriquées les unes dans les autres, souvent difficilement dissociables. Plus particulièrement, le récit biographique, en tant qu'activité discursive et que thème de discussion, renvoie aux procédés et aux produits d'un dire relatif à un parcours de vie, comme il renvoie aux ressources du je (le locuteur comme figure dans le discours) mises en œuvre pour l'accomplissement des activités langagières en cours. II peut être orienté vers un aspect particulier, les biographies langagières des élèves plurilingues, en ce sens qu'il renvoie essentiellement à des événements ou à des expériences personnelles, mais il peut aussi servir à référer à des éléments de l'identité d'un individu ou d'un collectif. Dans une perspective orientée vers la dimension narrative de l'entretien, nous pouvons distinguer trois instances du je dans le discours des élèves, en l'occurrence :

- le je-acteur permet de traiter le locuteur comme le personnage qui fait l'action dans le récit, dont on peut dire quelque chose et sur lequel on peut porter des jugements ;

- le je-narrateur, organisateur et évaluateur du récit langagier, permet de donner une perspective diachronique en faisant le lien entre le temps du récit et le temps de l'interaction;

- le je-narré, porteur des marques du récit, sert de support descriptif ou évaluatif pour positionner le locuteur qui l'emploie et rendre possible l'attestation des identités. 
Arrêtons-nous un peu sur ce dernier aspect, crucial pour nous, dans la mesure où l'interprétation des représentations de soi-même se construit, au plan énonciatif, par le choix de certaines désignations, comme de certaines formulations ou constructions syntaxiques (des séquences reliées syntaxiquement par des verbes ou noms métalinguistiques, par exemple). Être bilingue et/ou être plurilingue sont des profils langagiers, des catégories biographiques, des sphères identitaires disponibles dans le discours, mobilisées et interprétées en fonction de l'orientation des élèves vers ces identités dans la production de l'interaction. En ce sens, le fait d'avoir une identité revient, pour tout élève engagé dans la conversation, à son enchâssement dans une catégorie ayant des caractéristiques et des traits associés. Pour autant, le profil, comme processus, est une ressource discursive, pour reprendre les termes de Zimmerman (1998), qui oscille entre stabilisation et dynamisation: stabilisation dans le sens où il correspond en quelque sorte au caractère d'un individu, aux traits de sa personnalité, c'est-à-dire aux marques distinctives opératoires qui permettent de singulariser une personne comme individu, de l'identifier comme bilingue ou plurilingue, de le caractériser comme membre d'un ensemble; dynamisation dans le sens où il est rendu accessible par le récit, par l'activité de raconter son histoire à un moment donné à quelqu'un. Ce sont là autant de représentations qui pourront intervenir dans le sentiment d'identité en s'imbriquant étroitement aux processus identitaires et en les faisant converger.

7 Selon les contextes, ce ne sont pas les mêmes aspects de soi qui paraissent saillants ou utiles, et qui seront donc activés ou exprimés par l'individu. La question qui se pose est donc de savoir si, à travers des convergences et divergences des espaces des représentations en circulation, des déplacements des frontières de soi, on peut aller plus loin et dire que les élèves franco-allemands construisent des profils individuels et des territoires identitaires transportables qui connaissent inévitablement des changements et des évolutions en fonction de la trajectoire personnelle de chaque élève. Ce qui conduit à penser aussi que certaines représentations pourraient avoir un impact considérable sur les constructions et les dynamiques identitaires. Quoi qu'il en soit, ce qui prime pour nous, c'est que ces profils identitaires sont pluriels, hétérogènes et mouvants et ne surgissent pas par hasard.

Chaque élève a, on le sait, une histoire, sa propre histoire faite d'expériences personnelles, de socialisations et d'identifications (espérées ou imaginées, attestées ou décrites) à des valeurs, des langues, des normes, des modèles dans lesquels le sujet se reconnait, des groupes avec lesquels le sujet est en interaction. Les identités plurilingues, en particulier, sont loin de n'être que des instruments objectifs et socialement neutres, ne servant qu'à transmettre du sens, elles sont intimement liées à leurs utilisateurs. Cette caractérisation du profil identitaire, sous forme de continuum, à partir de la notion de subjectivité ouvre, à notre sens, une perspective intéressante pour l'analyse des représentations du plurilinguisme et pour l'appréhension de la singularité des trajectoires des élèves franco-allemands : elle concilie deux catégories que Locke tenait pour contraires l'une de l'autre, l'identité et la diversité. Ce constat nous semble d'autant plus important qu'il montre la nécessité de ne pas procéder à la définition des profils par des catégories fixées à l'avance, même si elles relèvent du sens commun.

Lüdi (1995 : 209) a écrit, à ce propos, que "la notion d'identité sert à mettre de l'ordre et de la permanence dans un domaine où tout semble a priori être en mouvement. D'abord - et tout en admettant qu'elle puisse subir des modifications plus ou moins profondes avec le temps-, l'identité sert à garantir que les 'je' successifs, que les différentes apparitions comme acteur dans des 
situations sociales sont bien des manifestations d'un seul et même individu". Posant comme préalable que l'interaction ne saurait être réduite à une alternance de représentations et de catégorisations successives autonomes, mais qu'elle se définit plutôt comme une activité sociale, productrice d'identités plurilingues, dynamiques, construites, négociées en permanence et conjointement par les locuteurs, nous voulons montrer que les représentations du plurilinguisme - y compris le poids des normes et des structures linguistiques de la (des) langue(s) première(s) - peuvent avoir des répercussions identitaires importantes et, de ce fait, déterminer les profils d'apprenants et les choix linguistiques en situation de contact de langues.

\title{
3. Être bilingue pour devenir plurilingue : images de soi dans le discours
}

Selon une belle formule de Hahn, il faudrait chercher à analyser

\begin{abstract}
die Selbstbesschreibung von Personen mittels 'partizipativer' Identitäten [...]. Man macht in allen Fällen eine Identität geltend, die man mit anderen gemeinsam hat. Zugleich aber aktiviert man eine Unterscheidung: Man identifiziert sich durch ein Merkmal oder eine ganze Klasse von Merkmalen, die andere - so wird jedenfalls unterstellt - nicht haben: Ich bin Mann und keine Frau, Katholik und nicht Protestant, Deutscher und nicht Franzose [...]. Die Identifikation, die hier vorgenommen wird, macht also einerseits den Anspruch auf eine Zugehörigkeit geltend und schließt gleichzeitig andere von dieser Zugehörigkeit aus (2000: 13) ${ }^{1}$.
\end{abstract}

Disons, en reformulant cette considération, que l'identité d'un individu vu comme locus et acteur social est caractérisée par deux dimensions, la première relevant de l'identité sociale (synchronique ou combinatoire), la seconde de l'identité personnelle (diachronique). Cette dichotomie a déjà fait l'objet d'investigations détaillées dans des perspectives théoriques diverses ( $c f$. Beacco et al, éd. 2005), que ce soit pour en relativiser les fondements ou pour en renforcer les effets. II n'est donc pas question de présenter ici dans toutes ses subtiles nuances cette problématique, mais plus modestement d'évoquer à grands traits quelques éléments qui renvoient à sa complexité constitutive. Car, dans le cas spécifique des entretiens biographiques, même si ces deux dimensions ont un statut heuristique et peuvent être distinguées pour des raisons analytiques, elles sont indissociables et présentes dans le discours par un travail sur la formulation et la reformulation des appartenances, sur les formes langagières attestant l'affiliation (inclusion versus exclusion) ou la participation d'un individu ou groupe d'individus à une communauté. Dans cette optique, nous pouvons avancer l'hypothèse selon laquelle l'identité sociale et l'identité personnelle de l'élève plurilingue (y compris l'identité située, historique, potentielle, imaginée ou hypothétique, projetée dans le futur, je suis bilingue et serai plurilingue en fin de scolarisation) ne font qu'une dans le cadre de leur accomplissement interactionnel dans la production conjointe du discours.

12 En même temps, les catégories (ou réseaux catégoriels) étant associées conventionnellement à des traits caractéristiques, à partir du lien entre prédicats et noms, le fait de savoir quelque chose sur une catégorie peut être utilisé efficacement pour décrire une personne ou l'identifier à un groupe par opposition à un autre (identifier une personne en tant qu'elle est cette personne et qu'elle se distingue d'une autre). Une fois la catégorisation opérée, la personne peut être vue comme appartenant à cette catégorie au sens où ce qu'on dit d'elle renvoie à ce que l'on peut dire de cette catégorie, et 
inversement. Deux questions se posent, à ce stade de notre réflexion, complémentaires l'une de l'autre: comment mettre en relief, dans notre analyse, la corrélation entre les représentations du plurilinguisme et les présentations identitaires ? Plus exactement, de quelle(s) modalité(s) de l'identité s'agit-il dans le discours biographique des élèves plurilingues? Nous tenterons de répondre à ces questions en mobilisant la fonction mimétique (mimèsis) de la représentation ${ }^{2}$ comme un des moyens permettant au locuteur de s'identifier, de se présenter et de se situer comme sujet et comme acteur, en rapport avec autrui : en tant que sujet, le locuteur se positionne par rapport à des valeurs et à des normes, en tant qu'acteur, il protège son image, sa "face"3 (au sens théâtral du terme) visà-vis de son interlocuteur. Considérons dans cette perspective un extrait d'entretien avec quatre élèves du lycée Sarrebruck.

1. SB-C. Ich sehe auch einen großen Unterschied zwischen Mehrsprachigkeit und Zweisprachigkeit. Zweisprachigkeit ist für mich, wenn man zwei Sprachen so beherrscht, dass man sich darin zuhause fühlt, dass man jederzeit wechseln kann, dass man praktisch gar nicht mehr richtig merkt, ob das Deutsch oder Französisch ist. Deswegen glaube ich auch, dass für jemanden, dessen Muttersprache Deutsch ist, es sehr schwierig ist, zweisprachig mit Französisch zu werden. Das ist fast unmöglich. Man kann das sehr gut beherrschen, im Endeffekt aber so richtig zweisprachig, habe ich das Gefühl, kann man nicht werden. Außer man lebt dann vielleicht für eine Zeit in Frankreich. Mehrsprachig kann man auf jeden Fall sein, wenn man diese Sprachen irgendwann spricht und versteht, aber das ist nicht perfekt. Mehrsprachig ist, wenn man z.B. Deutsch, Französisch, Englisch, Spanisch in seinem Repertoire hat oder so spricht und versteht, aber das ist etwas anderes als zweisprachig, finde ich.

2. SB-D. Dem stimme ich teilweise zu und widerspreche ich teilweise. Man muss es nicht unbedingt als Muttersprache haben, aber es ist trotzdem ein großer Unterschied ob man jetzt mehrere Sprachen kann oder nur zwei. Ich kenne es von mir selbst: Meine Eltern sind deutschsprachig und ich habe dann recht früh Französisch gelernt und mir wurde gesagt, dass ich kaum einen Akzent in Französisch habe. Viel später habe ich dann Englisch dazu gelernt und ich glaube, dass ich das viel besser kann als Französisch, dafür aber mit Akzent. Ein großes Problem hatte ich, als ich zu den zwei Sprachen später noch die dritte Sprache dazu bekam. Als ich in Amerika im Urlaub war und viel Englisch gesprochen habe, habe ich dann später versucht Französisch zu reden. Da habe ich dann die Sprachen verwechselt, z.B. englische Ausdrücke französisch ausgesprochen, in der Annahme es wären französische Wörter. Z.B. für die Münzen „un coin“ statt „a coin“. Ich denke, es wird irgendwann sehr schwer, wenn man verschiedene Sprachen spricht und es ist leicht, dass man diese Fremdsprachen dann irgendwie verwechselt.

3. SB-C. Ich fühle mich auch nicht als Zweisprachiger. Hier in der Schule kann man gut Französisch sprechen und man versteht auch sehr gut, aber man ist trotzdem nicht zweisprachig. Ich fühle mich eher mehrsprachig, aber wenn ich jetzt z.B. mehrere Wochen in Frankreich im Urlaub bin, dann fange ich an Französisch zu denken und dann halte ich mich schon eher für zweisprachig. Aber wie schon gesagt, nur in manchen Situationen.

4. SB-B. Natürlich bin ich damit völlig einverstanden und was mir öfters passiert ist, z.B. mit meiner Schwester, die ja wie ich auch zweisprachig ist, dass, wenn jemand kommt und z.B. Französisch nicht versteht und wir nicht wollen, dass er über ein Thema Bescheid weiß, dann benutzen wir natürlich die andere Sprache. Und das kommt nicht selten vor.

5. SB-E. Ich kann aus Erfahrung sagen, dass es nützlicher ist, schon in der Familie mit Sprachen anzufangen, dass man schon in jungen Jahren mit beiden Sprachen konfrontiert ist, damit man sich besser darin zurecht finden kann. Sehr wichtig ist dabei auch, dass man ständig mit der Sprache zu tun hat, nicht 
einige Jahre lang nur Deutsch spricht und dann Kontakt mit Franzosen hat. Dann verlernt man das sehr schnell wieder.

6. SB-E. Das hängt auch von der Kapazität des Einzelnen ab. Manche haben ein deutsches und ein französisches Elternteil und sind in keiner Sprache zuhause und haben keine richtige Muttersprache. Und andere kriegen das wieder ganz wunderbar hin, sind in beiden Sprachen perfekt, können gut schreiben und lesen. Ich weiß nicht, wovon es abhängt, vielleicht Intelligenzunterschiede oder Speicherunterschiede. Aber prinzipiell ist es am besten, wenn man es von Anfang an in der Familie lernt, denn so wie man das da lernen kann lernt man es nie wieder, finde ich zumindest. Also ich habe in den ersten vier Jahren kein Französisch gesprochen und merke das heute immer noch, obwohl ich ganz viel in Frankreich unterwegs bin und auch lange und trotzdem merke ich immer wieder, dass es nicht meine Muttersprache ist. Ich mag es und kann es gut, aber es ist nicht meine Muttersprache.

7. SB-C. Dem stimme ich zu, aber ich habe auch ältere Leute kennen gelernt, die später noch eine Sprache gelernt haben und da ist immer auch ein Akzent dabei. Ich denke, den bekommt man nur sehr schwer los, aber trotzdem können die Leute flüssig sprechen und reden. Ich kannte früher schon Leute im jüngeren Alter, die dann zweisprachig aufgewachsen sind und diese zwei Sprachen durcheinander gebracht haben. Die fingen mit einem deutschen Satz an und hörten mit Französisch auf. Denen viel es anscheinend schwer, die beiden Sprachen auseinander zu halten. Aber später habe ich diese Leute dann wieder gesehen und da ging das dann, die haben dann flüssig Deutsch und flüssig Französisch reden können, so dass das anscheinend später noch mal sortiert wird. Und deswegen denke ich, dass es am idealsten wäre, wenn man eine französischsprachige Mutter und einen deutschsprachigen Vater oder so was hat und die dann auch noch in einem anderen Land leben, so dass das Kind früh mit möglichst vielen Sprachen konfrontiert wird.

14 Lorsque nous analysons des représentations du plurilinguisme des élèves, nous sommes forcement confrontée à la circulation des mots et des discours, aux différentes formes de paroles rapportées, imaginées, empruntées ou même importées d'une communauté à l'autre (communauté éducative $\leftrightarrow$ enseignants $\leftrightarrow$ élèves, entre autres). Or, certains mots et certains énoncés sont porteurs de "mémoire sémantique" : comme le dit Bakhtine, ils sont "habités" par les sens et les contextes qu'ils ont déjà rencontrés, ils transportent en eux-mêmes les différentes acceptions dont ils se colorent à travers leurs voyages dans différentes communautés, dans un mouvement dialogique à la fois "intertextuel" et "interactionnel". À ces informations sémantiques, nombreuses et complexes, s'ajoutent celles qui sont fournies par l'interprétation des données grammaticales de surface (marques morphosyntaxiques, ordre des mots, désignations qualifiantes, etc.), celles qui viennent des inférences, et enfin celles qui sont apportées, à l'extérieur du langage, par la situation d'énonciation. Nous nous proposons, pour notre part, trois plans d'interprétation de ces informations : in fieri, in posse et in esse.

Sur le plan interactionnel (in fieri), les représentations identitaires et les présentations de soi se construisent progressivement au fur et à mesure de la rencontre. Ces représentations évoluent au cours de l'interaction et, comme tout discours argumentatif propice à l'émergence d'accords et de désaccords, les énoncés ne visent pas uniquement à faire connaître à l'autre une conviction, ils servent aussi à protéger ou à restaurer une image potentiellement déstabilisée à la suite d'une valeur ou d'une opinion non partagée. Pour interpréter ces énoncés, il convient de considérer que la présentation de soi émerge de la relation que le locuteur établit avec son interlocuteur, relation que l'on pourrait symboliser comme suit : - je (narrateur) $\neq t u$ (narrataire) $\neq i l$, autrui (actant) -. Cependant, 
tous les élèves n'adoptent pas les mêmes stratégies discursives pour accomplir leur mise en scène, le récit identitaire des élèves se fondant non seulement sur les représentations objectives, mais également sur des expériences vécues subjectives et des attentes d'ordre social. Pour illustrer cette idée, regardons d'abord la façon dont la structuration des énoncés, les enchaînements discursifs, concessifs ou expressifs et les formes de reprise de l'objet du discours témoignent de la construction des images et de la gestion des voix des locuteurs.

$\mathrm{Du}$ point de vue de l'organisation temporelle, le passage qui retient notre attention commence par l'intervention (1) de l'élève SB-C. Le locuteur exprime son opinion sur le plurilinguisme (thème) en la médiatisant sous la forme de l'image d'un tiers (man). Cette opération d'ancrage est essentielle, puisqu'elle garantit à l'interaction verbale à la fois sa cohésion, son intelligibilité et sa progression thématique, c'est-à-dire la "trame" de la discussion avec son réseau de repères énonciatifs, de variables référentielles et de procédés linguistiques. Les autres locuteurs confirment les réflexions proposées et identifient, sous forme d'assertions, plusieurs éléments (rhème) considérés comme pertinents dans le discours (ich sehe auch einen großen Unterschied, dem stimme ich teilweise $z \mathfrak{u}$ und widerspreche ich teilweise, also das mit dem Verwechseln, das kenne ich auch, meine Vorredner haben verstanden...so wie ich das sehe). Cet enchevêtrement possible des rhèmes nécessite une gestion d'ensemble articulée au but poursuivi. Nous distinguons donc trois phases dans la description de ces éléments, à savoir la situation initiale, la complication et la situation finale.

Les situations initiale et finale présentent et articulent des procès relativement stables ou équilibrés (Zweisprachigkeit ist für mich), alors que la complication se caractérise plutôt par des procès dynamiques ; l'activité discursive, étant mouvante, met en scène des points de vue différents. Par exemple, aux plans linguistique et relationnel, la complication consiste à marquer l'opposition entre une série d'éléments centraux posés en avant-plan, mis en évidence comme "figure » (présent de l'indicatif et du futur) et une autre série qui est de l'ordre du détail, posée en arrière-plan, constituant la scène ou le «fond» (passé composé, imparfait). Cette organisation est attestable dans des segments articulés entre eux par un procédé d'enchâssement syntaxique, auquel est parfois associée une opposition entre temps du verbe simples et composés, surtout lorsqu'il s'agit d'une relation d'antériorité relative. Pour prendre un exemple, citons les propos des élèves SB-C (1) et SB-D (2) : "Deswegen glaube ich auch, dass für jemanden, dessen Muttersprache Deutsch ist, es sehr schwierig ist, zweisprachig mit Französisch zu werden [...]. Deswegen glaube ich ist es schwierig, zweisprachig mit Französisch zu werden, aber es ist trotzdem ein großer Unterschied ob man jetzt mehrere Sprachen kann oder nur zwei (...) ich habe dann recht früh Französisch gelernt (...) viel später habe ich dann Englisch dazu gelernt (...) ein großes Problem hatte ich, als ich zu den zwei Sprachen später noch die dritte Sprache dazu bekam". Enfin, on notera que les éléments de référence temporelle peuvent être construits tout au long de l'interaction, manifestant des relations interactives de préalable et de succession, et qu'ils sont généralement explicités par des déictiques temporels, par des adverbes ou syntagmes à valeur temporelle (früh, später, selten, immer noch, wieder, nachher).

Sur le plan linguistique (in posse), nous proposons d'identifier trois niveaux précisant la nature et la configuration des segments compositionnels du discours, chaque niveau se définissant par des stratégies discursives spécifiques :

- les discours des élèves se produisent dans une situation de communication donnée qui donne lieu à des procédés de démonstration (problématiser), d'explication (se positionner) 
et de persuasion (prouver par des raisonnements successifs, des concessions ou des contradictions) ;

- le fait que l'entretien porte sur des objets de discours déterminés, à savoir la notion de plurilinguisme et la compétence plurilingue des élèves, renvoie à des savoirs conceptuels que les élèves doivent être en mesure de mobiliser et dont ils négocient la validité dans leurs productions discursives;

- le processus argumentatif obéit à certaines conditions de mise en scène discursive et tire sa force d'influence d'un certain nombre d'arguments interprétés à l'intérieur d'une situation donnée (à travers l'emploi des synonymes ou le recours à la notion de prototype, par exemple).

Si l'on essaie maintenant de préciser l'organisation séquentielle, nous distinguons deux éléments importants, les effets argumentatifs et les effets narratifs. Les effets argumentatifs, d'une part, se manifestent par la fréquence des connecteurs, des expressions modales, du lexique axiologique, ainsi que par la mise en place d'une configuration temporelle basée, on l'a vu, sur la récurrence du présent de l'indicatif, du futur et du passé composé Énonciativement, ceux-ci se trouvent renforcés par l'émergence de relations interactives génériques, telles que celles d'argument, de contre-argument, de reformulation ou de clarification, comme le montrent les séquences suivantes SB-C (7): "denen fiel es anscheinend schwer, die beiden Sprachen auseinander zu halten. Aber später habe ich diese Leute dann wieder gesehen und da ging das dann, die haben dann flüssig Deutsch und flüssig Französisch reden können, so dass das anscheinend später noch mal sortiert wird". Les effets narratifs, d'autre part, se manifestent dans les séquences narratives par des opérations de thématisation et des mises en relation. Dans ce dernier cas, trois possibilités se présentent : les séquences sont alternées, les séquences sont enchâssées et les séquences sont enchaînées.

Dans l'extrait, l'alternance est la forme la plus complexe de combinaison séquentielle, présentant parallèlement deux thèmes, comme en témoigne la séquence suivante (4) :

so hängt das auch von der jeweiligen Situationen ab. - Natürlich bin ich damit völlig einverstanden und was mir öfters passiert ist, z.B. mit meiner Schwester, die ja wie ich auch zweisprachig, ist, dass, wenn jemand kommt und z.B. Französisch nicht versteht und wir nicht wollen, dass er über ein Thema Bescheid weiß, dann benutzen wir natürlich die andere Sprache. Und das kommt nicht selten vor.

21 Plus fréquent que l'alternance, l'enchâssement des séquences met en scène des personnages et des voix différents, notamment lorsque l'élève est amené à faire des autoattributions à propos d'évenements positifs, comme l'atteste la séquence suivante (5):

Ich kann aus Erfahrung sagen, dass es nützlicher ist, schon in der Familie mit Sprachen anzufangen, dass man schon in jungen Jahren mit beiden Sprachen konfrontiert ist, damit man sich besser darin zurecht finden kann. Sehr wichtig ist dabei auch, dass man ständig mit der Sprache zu tun hat, nicht einige Jahre lang nur Deutsch spricht und dann Kontakt mit Franzosen hat. Dann verlernt man das sehr schnell wieder.

Pour terminer, on peut ajouter que l'enchaînement articule et organise des séquences par comparaison et juxtaposition, permettant de distinguer entre des composantes sociales qui constituent l'identité sociale de l'élève et les composantes constituant son identité personnelle, comme le montre la séquence suivante (6) :

Das hängt auch von der Kapazität des Einzelnen ab. Manche haben ein deutsches und ein französisches Elternteil und sind in keiner Sprache zuhause und haben keine richtige Muttersprache. Und andere kriegen das wieder ganz wunderbar hin, 
sind in beiden Sprachen perfekt, können gut schreiben und lesen. (...). Aber prinzipiell ist es am besten, wenn man es von Anfang an in der Familie lernt, denn so wie man das da lernen kann lernt man es nie wieder, finde ich zumindest". En articulant ces différents niveaux d'analyse, la présentation de soi peut s'envisager à la fois comme une structure stable, un filtre cognitif modulant la perception de toute information contradictoire et comme une structure souple et complexe en évolution constante, suscitant diverses strates d'interprétation qui sont autant de clés pour accéder à ce que les élèves veulent montrer d'eux-mêmes. rapports que chaque interactant établit avec les autres, dès lors qu'il identifie des individus bilingues ou plurilingues, et qu'il établit des liens avec son passé, avec le présent et avec le futur. Se reconnaitre comme plurilingue ou bilingue active un réseau de représentations, une constellation de valeurs de référence et d'attentes schématiques auxquelles le locuteur veut montrer qu'il s'associe. Ces représentations peuvent jouer le rôle de marqueur identitaire en suscitant des prises de position communes ou divergentes à propos de l'objet du discours. Inversement, elles peuvent être déterminées par des identités et, dans ce cas, elles peuvent jouer un rôle d'affirmation de la distinctivité d'un groupe. Enfin, elles peuvent être des régulateurs identitaires en organisant le processus d'interaction : plus un groupe est perçu comme homogène, plus les individus s'estiment en mesure d'affirmer leur(s) identité(s) et de justifier leurs comportements langagiers.

Partant de l'idée selon laquelle tout individu est caractérisé, d'un côté, par des traits d'ordre social qui signalent son appartenance à des groupes ou catégories et, de l'autre, par des traits d'ordre personnel, des attributs plus spécifiques de l'individu, plus idiosyncrasiques, nous proposons de distinguer trois niveaux référentiels qui constituent, en quelque sorte, les cadres de construction de la représentation identitaire chez les élèves plurilingues : le premier est le niveau hyper ordonné, où l'élève se conçoit comme acteur social et réalise des comparaisons à travers des assimilations et des différenciations (réelles ou symboliques) entre soi et autrui. Le deuxième niveau est dit intermédiaire ou méso, où l'élève, se catégorisant comme membre d'un groupe, réalise des comparaisons intergroupes (l'exogroupe). Et le troisième niveau est dit hypo ordonné, où l'élève, se concevant comme une personne singulière, unique et distincte, réalise des comparaisons entre lui-même et autrui, à l'intérieur du groupe d'appartenance (l'endo groupe). Ainsi, nous faisons l'hypothèse que les degrés variables d'implication des représentations dans la construction identitaire peuvent être traités à l'aide des dimensions opératoires suivantes, qui, comme le montre le schéma ci-dessous, s'imbriquent les unes dans les autres : 
Figure 1 : Cadres de construction de la représentation identitaire

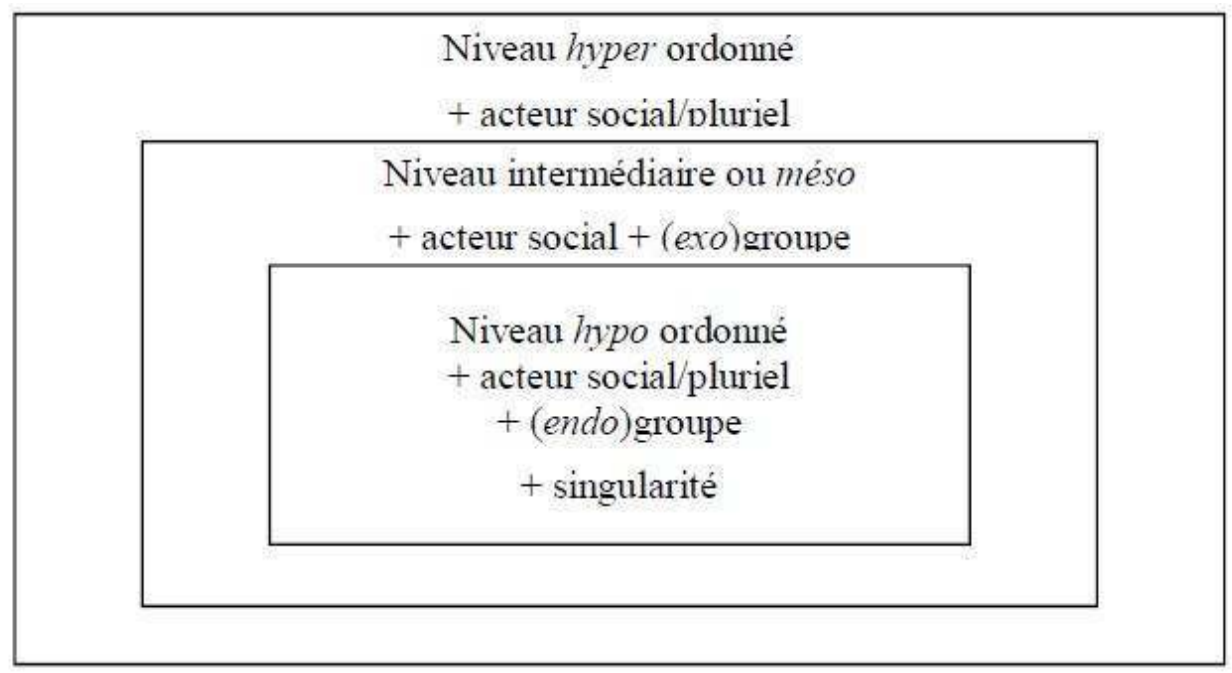
intragroupe peut produire des effets de contraste plus ou moins forts, elle peut conduire l'élève à se percevoir comme très différent des membres de son propre groupe (différenciation forte) ou comme très semblable (différenciation faible). De la même façon, la comparaison intergroupe entraînera des effets de différenciation variables par rapport aux membres de l'exogroupe. Pour s'en convaincre, il suffit de considérer l'image qui se dégage à travers le discours des élèves, image (ou plutôt images multiples) que les élèves cherchent à donner d'eux-mêmes. Pour prendre un exemple, citons les propos de l'élève SB-C (3) :

Ich fühle mich auch nicht als Zweisprachiger. Hier in der Schule kann man gut Französisch sprechen und man versteht auch sehr gut, aber man ist trotzdem nicht zweisprachig. Ich fühle mich eher mehrsprachig, aber wenn ich jetzt z.B. mehrere Wochen in Frankreich im Urlaub bin, dann fange ich an Französisch zu denken und dann halte ich mich schon eher für zweisprachig. Aber wie schon gesagt, nur in manchen Situationen.

À première vue, et d'un point de vue naif, on pourrait être tenté d'opposer d'un côté le pôle bilingue, de l'autre le pôle plurilingue à l'image de ce qui a longtemps dominé les travaux, monolingue versus bilingue. Mais, à y regarder de près, on constate que les représentations des élèves franco-allemands sont beaucoup plus nuancées que ne le laisserait penser une telle présentation schématique. Les frontières (mouvantes, évolutives, symboliques ?) entre les deux restent difficiles à établir et la prise en compte des profils et des identités plurielles des élèves ne relève certainement pas exclusivement de l'un des deux pôles. Les représentations apparaissant comme autant de portraits souvent flous, parfois contradictoires mais toujours discernables des profils des élèves, nous proposons dès lors de les considérer en termes de stratégies et de (en)jeux identitaires au travers des comparaisons et des différenciations que l'élève établit entre représentations de Soi et représentations sociales. Et rien n'interdit de penser que ces différents cadres de représentation s'imbriquent les uns aux autres en des configurations multiples, contribuant ensemble au sentiment d'identité. Dans cette perspective, qu'il s'agisse de notions, d'opinions ou de valeurs, les représentations disponibles en l'élève sont toujours dialogiques et interactives, en ce sens qu'elles intègrent les représentations des Autres, qu'elles continuent de s'y confronter, de les négocier dans la comparaison à autrui. 
En guise donc de conclusion, citons Claire Kramsch (2008: 322): "si en effet, les représentations sont 'acte social' (...), elles impliquent une puissance d'agir qui vient (...) d'une capacité à comprendre les discours qui forment aussi bien l'Autre que le Soi".

\section{Conclusion}

27 Sur la base de propositions émanant tantôt de l'analyse du discours, tantôt de la didactique du plurilinguisme, nous avons privilégié deux entrées dans notre analyse. Reprenant à Mondada \& Py (1994) l'hypothèse selon laquelle la notion de profil d'apprenant ne saurait être considérée de manière isolée et abstraite, nous avons envisagé, d'un côté, l'apprentissage des langues dans sa dimension diachronique comme un parcours construit tout au long de la vie, prenant en considération l'histoire et le vécu langagiers de l'élève en dehors de l'école ou avant celle-ci, c'est-à-dire le capital de relations à l'altérité linguistique déjà-là ou en voie de constitution des apprenants, altérité exploitée comme contraste entre catégorisations différentes, ou comme relais dans la pluralité des contacts de langues. D'autre part, nous avons adopté une perspective synchronique pour aborder, au moyen de l'analyse des entretiens, le répertoire langagier à un moment précis de la vie d'un apprenant. Ainsi, l'accent est mis à la fois sur l'identité actuelle et sur les identités possibles, à entendre, selon nous, comme les projections par l'individu de ce qu'il veut être (ou ne pas être), l'objectif étant de répondre aux questions suivantes : comment les biographies langagières, auxquelles on accède par le biais de l'analyse des récits, mettent en lumière différents parcours de construction identitaire, et plus particulièrement comment les expériences vécues d'appropriation des langues, à l'école et en dehors de celle-ci, sont susceptibles d'enrichir ou de complexifier les représentations et les pratiques langagières de l'apprenant tout au long de sa vie?

L'analyse a montré que l'identité plurilingue des élèves se construit dans la durée avec des périodes de latence ou de réactivation des diverses ressources linguistiques en fonction des références identitaires, des schèmes culturels et des interlocuteurs variables. La durée ne garantit pas un développement linéaire de cette compétence, comme elle ne contribue pas à la valorisation systématique de toutes ses formes. En effet, l'identité se révèle, dans le discours, comme composite et complexe à des degrés divers, construite différemment pour chaque élève en fonction des contextes sociaux, des espaces de socialisation, des réseaux relationnels et des communautés langagières. En ce sens, elle est constituée de différentes ressources et habilités, sociales et linguistiques, de strates diverses et de nœuds de relations à des sphères d'appartenances multiples, la coupure n'étant jamais radicale, aucune n'étant réellement exclue ni toutes jamais simultanément visibles dans un contexte particulier et dans une situation de communication donnée. Grâce à cette complexité, le plurilinguisme des élèves franco-allemands articule à la fois le social et l'individuel, la singularité et la pluralité, le linguistique et le culturel, la communication et la médiation culturelle. 


\section{BIBLIOGRAPHIE}

Beacco, J.-L., Chiss, J.-L., Cicurel, F. \& Véronique, D. (dir.) (2005). Les cultures éducatives et linguistiques dans l'enseignement des langues. Paris : Presses Universitaires de France.

Castellotti, V. \& Moore, D. (2002). Représentations sociales des langues et enseignements. Division des politiques linguistiques. Strasbourg : Conseil de l'Europe.

De Florio-Hansen, I. \& Hu, A. (éds.). (2007). Plurilingualität und Identität. Zur Selbst- und Fremdwahrnehmung mehrsprachiger Menschen. Tübingen : Stauffenburg.

Deschamps, J.-C. \& Moliner, P. (2008). L’identité en psychologie sociale. Paris : Armand Colin.

Goffman, E. (1974). Les rites de l'interaction. Paris : Minuit.

Goffman, E. (1959, 1973 trad. française) : La mise en scène de la vie quotidienne. Tome I, Paris: Minuit.

Hahn, A. (2000). Konstruktionen des Selbst, der Welt und der Geschichte. Frankfurt am Main :

Suhrkamp.

Kramsch, C. (2008). "Voix et contrevoix : l'expression de soi à travers la langue de l'autre". In G. Zarate, G., Lévy, D. \& Kramsch, C. (dir). Le précis du plurilinguisme et du pluriculturalisme., pp. 35-38. Paris : Éditions des archives contemporaines.

Lüdi, G. \& Py, b. (éds). (1995). Changement de langage et langage du changement. Lausanne : L'Age d'Homme.

Mondada, L. \& Py, B. (1994). "Vers une redéfinition interactionnelle de la notion d'apprenant", Actes du 9e Colloque Acquisition des Langues : Perspectives et Recherches, St.-Etienne, 13-15.5.1993, StEtienne : Presses de l'Université de Saint-Etienne, pp. 381-396.

Pepin, N. (2007). Identités fragmentées. Eléments pour une grammaire de l'identité. Berne: Peter Lang. Zimmerman, D. (1998). "Identity, context and interaction". In Antaki Ch. \& Widdicombe, S. (ed). Identities in talk. London : Sage, pp. 87-106.

\section{ANNEXES}

Annexe 1 Traduction en français de l'extrait

1. SB-C. Je vois aussi une grande différence entre plurilinguisme et bilinguisme. Être bilingue c'est pour moi lorsque quelqu'un maîtrise deux langues de sorte qu'il se sent chez soi dans les deux langues, que quelqu'un peut alterner les langues à n'importe quel moment, que quelqu'un ne se rend pas compte s'il s'agit de l'allemand ou du français. C'est la raison pour laquelle je crois que, pour quelqu'un dont la langue maternelle est l'allemand, il est très difficile de devenir bilingue en apprenant le français. C'est presque impossible. Quelqu'un peut le maîtriser très bien, mais en fin de compte devenir vraiment bilingue, je ne pense pas que ce soit possible. Sauf si quelqu'un vit pendant un certain temps en France. Plurilingue, quelqu'un peut le devenir de toute manière, lorsqu'il parle et comprend des langues, mais ne les maîtrise pas parfaitement. Plurilingue est quelqu'un 
qui a, par exemple, l'allemand, le français, l'anglais, l'espagnol dans son répertoire ou qui les parle et les comprend, mais c'est différent d'être bilingue, je trouve

2. SB-D. Je suis à la fois d'accord et pas d'accord. Elles ne devraient pas être absolument des langues maternelles, mais ça fait quand même une grande différence si quelqu'un connaît plusieurs langues ou seulement deux. Je parle de ma propre expérience : mes parents sont des germanophones et j'ai appris très tôt le français et ils m'ont dit que je n'avais presque pas d'accent en français. Beaucoup plus tard, j'ai appris aussi l'anglais et je crois que je le connais beaucoup mieux que le français, mais par contre avec un certain accent. J'avais un gros problème quand j'ai ajouté aux deux langues une troisième. Lorsque j'étais aux Etats-Unis en vacances, j'ai beaucoup parlé anglais ; j'ai essayé par la suite de parler français. Alors, j'ai mélangé les langues, par exemple des expressions anglaises prononcées comme en français, supposant qu'il s'agissait de mots français. Par exemple, pour les monnaies, "un coin" au lieu de "a coin". Je crois que ça devient, à un moment donné, très difficile, lorsque quelqu'un parle des langues différentes; ce n'est pas facile et le risque existe de mélanger, à un moment donné, ces langues étrangères.

3. SB-C. Je ne me sens pas non plus bilingue. Ici à l'école on peut très bien parler français et on comprend très bien mais on n'est quand même pas bilingue. Je me sens plutôt plurilingue, mais maintenant si je passe plusieurs semaines en vacances en France, je commence à penser en français et à ce moment-là je me considère comme plutôt bilingue. Mais comme on l'a déjà dit, seulement dans certaines situations.

4. SB-B. Bien évidemment, je suis entièrement d'accord et il m'est arrivé souvent, par exemple, avec ma sœur qui est aussi bilingue que moi, que, lorsque quelqu'un arrive, qu'il ne comprend pas par exemple le français, et qu'on ne veut pas qu'il comprenne le thème de notre discussion, d'employer naturellement l'autre langue. Et cela n'est pas rare.

5. SB-E. Je peux dire à partir de ma propre expérience, qu'il est plus utile de commencer déjà en famille avec les langues, qu'il est plus facile de se sentir à l'aise lorsqu'on y est confronté dès son plus jeune âge. II est aussi important d'être en contact permanent avec la langue ; pas seulement quelques années à ne parler qu'allemand et après chercher le contact avec les Français. On l'oublie après très rapidement.

6. SB-E. Cela dépend aussi des compétences de chacun. Certains ont des parents allemand et français et ils ne se sentent chez eux dans aucune langue et n'ont aucune vraie langue maternelle. Et d'autres arrivent à maîtriser la situation, sont parfaits dans les deux langues, peuvent bien écrire et bien lire. Je ne sais pas de quoi cela dépend, peut-être de différences intellectuelles ou de différentes capacités de mémoriser. Mais, en général, il vaut mieux que quelqu'un apprenne les langues dès le début en famille, parce que la manière dont on les apprend en famille on se retrouve plus ailleurs, je crois en tout cas. Donc, moi je n'ai pas parlé le français pendant quatre ans au début, et je le remarque encore aujourd'hui, même si je suis souvent en France et pour une longue durée ; quand même je remarque toujours que ce n'est pas ma langue maternelle. J'aime beaucoup [le français] et je le connais bien, mais ce n'est pas ma langue maternelle.

7. SB-C. Je suis d'accord, mais j'ai vu aussi des personnes âgées qui ont appris tard une langue et qui ont toujours un accent. Je crois que l'on peut difficilement s'en débarrasser, mais malgré ça, les gens peuvent parler et discuter couramment. Je connaissais auparavant des jeunes qui ont grandi bilingues et qui ont mélangé les langues. IIs commençaient leur phrase en allemand et ils la finissaient en français. C'était 
apparemment difficile pour eux de séparer les deux langues. Mais je les ai vus plus tard et alors ça y était, ils parlaient couramment l'allemand et le français, comme si, avec le temps, il était possible de séparer les langues. Et pour cette raison, je pense que l'idéal serait d'avoir une mère française et un père allemand ou quelque chose comme ça, et de vivre dans un autre pays afin d'être confronté tôt avec le plus grand nombre de langues.

\section{NOTES}

1. Traduit en français par nos soins: "[il faudrait chercher à analyser] l'auto-description des personnes à travers la notion d'identité participative. On affirme dans toutes les situations une identité, que l'on partage avec les autres. Or, en même temps, on affirme aussi notre différence. On s'identifie à travers une caractéristique ou une catégorie de caractéristiques, que les autres n'ont pas, du moins c'est ce que l'on prétend. Je suis un homme et pas une femme, je suis catholique et pas protestant, je suis allemand et pas français [...]. L'identification qui est mise en scène ici permet l'appartenance à un groupe tout en excluant d'autres groupes de cette appartenance".

2. L'identité (être semblable) est ainsi envisagée avec son revers, l'altérité (être différent).

3. Pour Goffman, tout individu (tout groupe) possède à la fois une face positive, la face d'ouverture vers l'Autre, et une face négative, la face de protection de son identité, de son territoire (dans la terminologie de l'auteur). Les termes positif et négatif de ces faces doivent être entendus au sens photographique comme la pellicule négative et positive d'une même identité, le recto et le verso d'un même être. Dans cette optique, les travaux de Goffman reconnaissent la relativité des normes et la mouvance des identités, mais en focalisant sur les identités stigmatisées ou négatives, ils mettent l'accent sur la faculté, dont dispose l'acteur, de se voir en train de jouer un rôle (ou une identité), c'est-à-dire qu'ils soulignent la distance existante entre le Moi et l'identité. Mais peut-on vraiment concevoir le Moi comme une sorte de noyau profond de la personne, noyau qui peut s'occulter, lors de certaines interactions sociales, derrière l'expression des rôles que le Moi est amené à assumer? Goffman (1974: 9) a écrit : "on peut définir le terme de face comme étant la valeur sociale positive qu'une personne revendique effectivement à travers la ligne d'action que les autres supposent qu'elle a adoptée au cours d'un contact particulier".

4. Afin de respecter l'anonymat des élèves interviewés, nous les notons par des lettres. Nous mettons en gras les passages les plus intéressants pour l'analyse. Le lecteur trouvera les traductions en français à la fin de l'article.

\section{RÉSUMÉS}

Notre étude s'inscrit dans la ligne des questionnements en linguistique et en didactique des langues et porte sur les conditions dans lesquelles s'opère la construction de l'identité plurilingue chez des apprenants placés, du fait de leur biographie langagière ou de leur mode de scolarisation, dans un établissement institutionnellement valorisant et réputé, tel que le lycée franco-allemand de Sarrebruck. La recherche s'intéresse plus particulièrement aux apports réciproques des représentations sociales du bi-/plurilinguisme et des contextes d'acquisition et interroge les conditions dans lesquelles les corpus recueillis permettent de constater ou d'inférer 
des transformations en cours dans les représentations des apprenants, que ces représentations soient relatives aux langues et à leur apprentissage ou qu'elles relèvent de la construction des connaissances.

Our research is in the field of linguistics and language didactics. It concerns the conditions for building multiple language identities in learners who, due to their language biography or the educational system, are studying in a prestigious institutional school environment, such as the French-German school of Saarbrücken. In particular, the research examines the reciprocal interaction between social representations of bi-/plurilingualism and contexts of acquisition. We review the conditions in which the data collected led us to conclude or to infer changes in learners' representations related to the languages and their language-learning or to their accumulation of knowledge.

\section{INDEX}

Mots-clés : représentations identitaires, plurilinguisme, trajectoires, discours

Keywords : representations of identity, plurilingualism, trajectories, discourse

\section{AUTEUR}

\section{SOFIA STRATILAKI}

Université Sorbonne Nouvelle - Paris 3, DILTEC 